\section{ROBUST: Lenalidomide-R-CHOP versus placebo-R-CHOP in previously untreated ABC-type diffuse large B-cell lymphoma}

\author{
Grzegorz S Nowakowski, ${ }^{* 1}$ Annalisa Chiappella², Thomas E Witzig', Michele Spina ${ }^{3}$, \\ Randy D Gascoyne ${ }^{4}$, Lei Zhang ${ }^{5}$, Jocelyne Flament ${ }^{5}$, Jacqueline Repici ${ }^{5}$ \\ \& Umberto Vitolo ${ }^{2}$
}

Activated B-cell-like $(A B C)$ diffuse large B-cell lymphoma (DLBCL), the major constituent of nongerminal center $B$-cell-like (non-GCB) DLBCL, is associated with poorer survival outcomes than GCB-type DLBCL. In Phase II studies, lenalidomide combined with R-CHOP ( $\mathrm{R}^{2}-\mathrm{CHOP}$ ) improved outcomes relative to historical $\mathrm{R}-\mathrm{CHOP}$ in newly diagnosed $\mathrm{DLBCL}$, particularly in non-GCB cases. ROBUST (CC-5013-DLC-002) is a randomized, double-blind, global, Phase III study of oral lenalidomide ( $15 \mathrm{mg}$, days $1-14$ ) plus R-CHOP $21 \times 6$ versus placebo-RCHOP21 $\times 6$ in patients with previously untreated ABC-type DLBCL. Subtyping is done within 3 calendar days by central laboratory gene-expression profiling of formalin-fixed paraffinembedded biopsy tissue. The primary end point is progression-free survival. Secondary end points include response rates, overall survival and health-related quality of life.

First draft submitted: 18 March 2016; Accepted for publication: 5 April 2016; Published online: 18 April 2016

Diffuse large B-cell lymphoma (DLBCL) is the most common WHO subclassification of B-cell non-Hodgkin lymphoma (NHL), constituting 30-40\% of all new cases [1]. Advances in chemoimmunotherapy have included the combination of rituximab with cyclophosphamide, doxorubicin, vincristine and prednisone (R-CHOP) [2], the regimen most frequently used in fit patients with advanced disease. Although this regimen has resulted in substantial improvements in cure rate, approximately $40 \%$ of patients with DLBCL will relapse, most of whom will ultimately succumb to the disease [3]. The need for improved therapeutic options therefore remains.

\section{DLBCL subtypes}

The heterogeneity and highly variable clinical course of DLBCL is suggestive of several different phenotypes with divergent responses to therapy. Efforts to define subtypes based on morphology have proven problematic $[4,5]$. Some prognostic factors, such as the nuclear protein Ki-67, a marker of tumor cell proliferation, predicted survival with the standard regimens of $\mathrm{CHOP}[6]$ and, later, R-CHOP [7], but the molecular basis for this remained unknown. Clinical parameter-based models such as the International Prognostic Index (IPI) [8], the revised IPI (R-IPI) [9] and the National Comprehensive Cancer Network IPI (NCCN-IPI) [10] separate patients into distinct prognostic subgroups and have been available for some years; however, the cellular and molecular differences

'Division of Hematology, Mayo Clinic, Rochester, MN 55902, USA

2Division of Hematology, Città della Salute e della Scienza Hospital \& University, Torino, Italy

${ }^{3}$ Division of Medical Oncology, National Cancer Institute, Aviano, Italy

${ }^{4}$ Department of Pathology \& Lymphoid Cancer Research, BC Cancer Agency \& BC Cancer Research Centre, Vancouver, BC, Canada

${ }^{5}$ Celgene Corporation, Summit, NJ 07901, USA

*Author for correspondence: Tel.: +1 507284 2511; Fax: +1 597266 4972; Nowakowski.Grzegorz@mayo.edu

\section{KEYWORDS}

- chemotherapy

- hematologic/lymphoma

- molecular oncology
Future
Medicine $\mathrm{fS}$ 
underlying these empirical categories were unclear, and risk scores were of limited utility in stratifying patients for therapeutic trials [11]. The possibility that clinical prognostic categories may be proxies for DLBCL subtypes possessing distinct genetic profiles led to an exploration of gene expression in lymph node biopsy samples from patients with de novo DLBCL.

Gene-expression profiling (GEP) using microarrays originally identified two major distinct DLBCL subtypes with expression patterns consistent with different stages of B-cell differentiation: germinal center B-cell-like (GCB) and activated B-cell-like (ABC) [12]. These subtypes differed in the expression of hundreds of genes. DLBCL patients with ABC subtype were found to have significantly ( $\mathrm{p}<0.01)$ shorter survival, with a 5-year overall survival (OS) rate of only 16 versus $76 \%$ for those with GCB subtype in the CHOP era [12]. Classification by these cellof-origin (COO) subtypes proved distinct from IPI risk groups, in fact adding to the prognostic value of IPI, with the ABC subtype predicting significantly $(\mathrm{p}<0.05)$ worse OS than the GCB subtype among all low-risk IPI patients. Subsequent studies have borne out the poorer survival associated with the ABC subtype, with 5 -year OS rates of 60 and 35\% in GCB and ABC subtype first-line DLBCL, respectively [13]. Further biological studies revealed different somatic mutation profiles and different signaling pathways, further supporting the notion that $\mathrm{ABC}$ and GCB DLBCL are indeed distinct biological subtypes of DLBCL [14].

Additional GEP studies identified a third DLBCL subtype that was neither ABC nor GCB [13]. This heterogeneous subtype was originally referred to as 'type 3' but later would simply be called 'unclassifiable'.

Although genome-wide GEP represented the 'gold standard' of DLBCL subtyping methods, as originally performed it was complex, expensive, time consuming and required fresh or frozen tissue, all of which made it impractical for routine clinical use. Immunohistochemistry (IHC) using formalin-fixed, paraffin-embedded (FFPE) tissue and as few as three immunostains proved more feasible [15] and provided an alternative to GEP. However, IHC at best defines only GCB and non-GCB subtypes and variably assigns the unclassifiable subtype to either category. In addition, while IHC performs relatively well in experienced hands, it is subject to technical and interpretative problems, with highly variable results for interlaboratory comparisons [16]. One study comparing IHC versus GEP subtyping found an IHC misclassification rate of between 30 and $60 \%$ for the GCB subset, with a resulting loss of survival prognostic ability [17]. However, despite the considerable loss of precision compared with GEP, the relative convenience and availability of IHC led to its widespread adoption for DLBCL subtyping in the clinic.

Recently, a novel GEP assay for DLBCL subtyping that uses FFPE tissue samples, Lymph2Cx, was developed [18]. Using the commercial NanoString ${ }^{\circledR}$ nCounter ${ }^{\circledR}$ Analysis System (NanoString Technologies, WA, USA) and starting with a set of 93 genes identified as differentiating between $\mathrm{ABC}$ and $\mathrm{GCB}$ subtypes [19], investigators determined that a set of 15 core genes, along with five housekeeping genes, predicted $\mathrm{COO}$ as determined by the 'gold standard' of full GEP. The resulting 20 -gene assay was validated in an independent cohort of FFPE tissue samples. The subtype misassignment rate was only $2 \%$, which compares favorably to the corresponding $6-17 \%$ rates reported for IHC-based methods (Figure 1) [18]. Importantly, concordance of COO identification between two independent laboratories was $>95 \%$. The Lymph2Cx assay was further studied in a large population-based cohort of patients treated with R-CHOP and was shown to retain its prognostic value independent of IPI score [20]. Use of this assay in clinical trials such as the ROBUST study described herein is expected to increase the accuracy of subtype classification, an important step in better matching patient to treatment, the goal of precision medicine [21].

\section{Lenalidomide in DLBCL}

Lenalidomide is an oral immunomodulatory drug that has direct antineoplastic activity, as well as indirect effects mediated via immune cells present in the tumor microenvironment [22] Lenalidomide targets the E3 ubiquitin ligase cereblon [23]. In preclinical studies lenalidomide exhibits synergy with rituximab [24,25], and clinical experience has associated single-agent and combination lenalidomide with durable responses in multiple types of both indolent and aggressive relapsed/refractory NHL. Lenalidomide is approved by the US FDA for multiple myeloma in combination with dexamethasone and for transfusion-dependent anemia due to low- or intermediate-1-risk myelodysplastic syndromes associated with a deletion $5 \mathrm{q}$ 


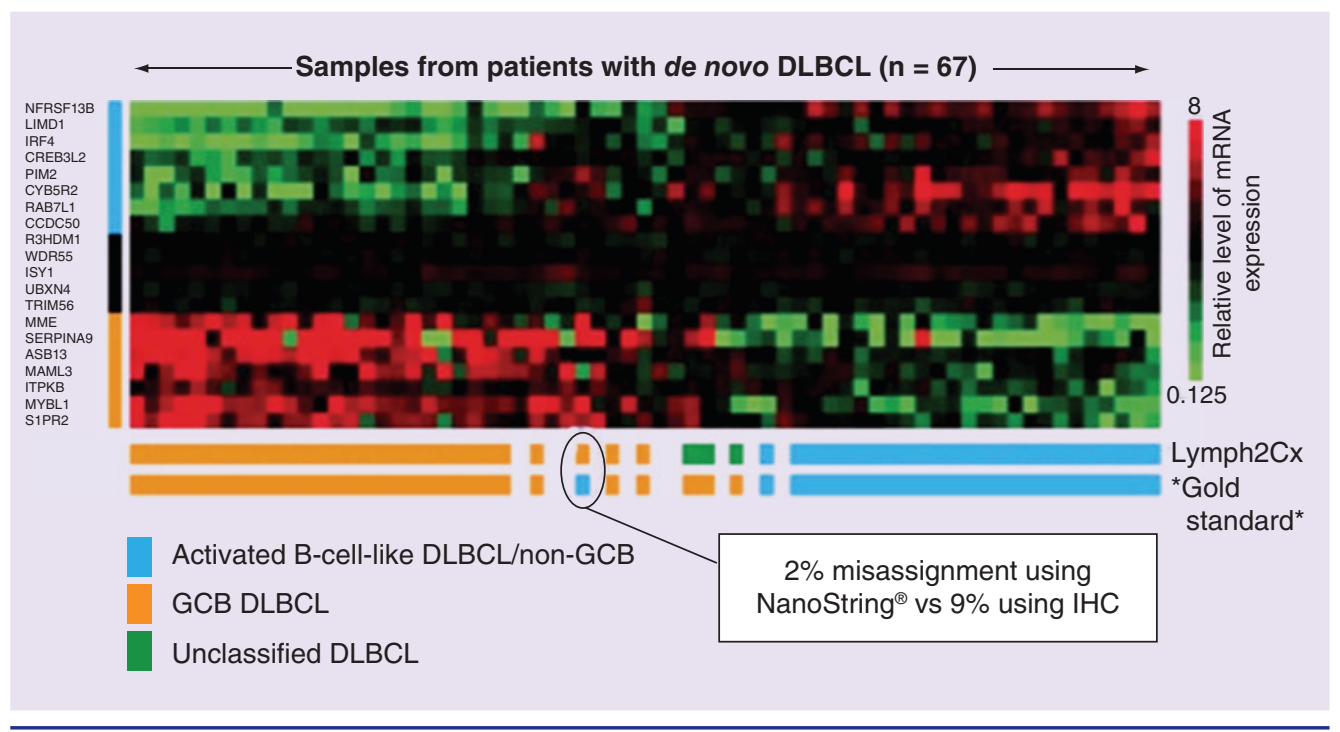

Figure 1. NanoString gene-expression profiling using the Lymph2Cx assay.

DLBCL: Diffuse large B-cell lymphoma; GCB: Germinal center B cell; IHC: Immunohistochemistry.

Republished with permission from [18] $\odot$ Clearance Center, Inc.

abnormality. In mantle cell lymphoma, lenalidomide is FDA approved for relapsed or progressed disease after two prior therapies, one of which included bortezomib [26].

In a xenograft model of DLBCL, lenalidomide had a more pronounced effect on $A B C$ subtype disease than on the non-ABC subtype [27], which was associated with downregulation of the transcription factor IRF4, a downstream target of cereblon, with a consequent downregulation of B-cell receptor-dependent NF- $\kappa \mathrm{B}$. Two clinical studies explored the safety and activity of lenalidomide in relapsed/refractory aggressive NHL, including DLBCL [28,29]. Patients were treated with oral lenalidomide ( $25 \mathrm{mg}$ daily) on days 1-21 of each 28-day cycle. COO subtyping was not performed. Pooled analysis of the 134 patients with DLBCL from these trials (median age 66 years, median of three prior regimens) revealed an overall response rate (ORR) of $26 \%$, including 9\% achieving complete response (CR)/CR unconfirmed. The median duration of response was 6 months. The safety profile of lenalidomide was consistent with that observed in other studies, the most frequent grade 3 or 4 events being reversible neutropenia (22 and $13 \%)$, thrombocytopenia (16 and 5\%) and anemia (6 and $0.7 \%$ ) [30].

In a retrospective review of 40 patients with relapsed/refractory DLBCL who were treated with lenalidomide and who had subtype assigned by IHC, a significantly improved response
(53 vs $9 \%, \mathrm{p}=0.006$ ), as well as significantly better median progression-free survival (PFS; 6.2 vs 1.7 months, $\mathrm{p}=0.004$ ), was reported in the non-GCB versus GCB subtype [31]. Thus, clinical evidence supported further investigation of lenalidomide in relapsed/refractory DLBCL.

The prospective DLC-001 trial randomized 102 patients with relapsed/refractory DLBCL stratified by COO subtype as determined by IHC using the Hans algorithm [15] to receive either oral lenalidomide (25 mg daily, days 1-21 of each 28-day cycle) or investigator's choice monotherapy consisting of single-agent gemcitabine, rituximab, etoposide or oxaliplatin [32]. In this third plus-line population, lenalidomide was associated with improved PFS relative to investigator's choice in the overall patient population (13.6 vs 7.9 weeks, $\mathrm{p}=0.041$ ). Among lenalidomide-treated patients with DLBCL classified as GCB or non-GCB, ORR was similar (26 vs $29 \%$ ), but PFS (10.1 vs 15.1 weeks) and OS ( 30.0 vs 32.3 weeks) were greater in the nonGCB group. Exploratory analysis using microarray GEP found significant efficacy differences between the more precisely defined $\mathrm{ABC}$ and GCB groups. Lenalidomide-treated patients with the ABC subtype had improvement in ORR ( 45 vs $21 \%$ ), median PFS (82.0 vs 13.2 weeks) and median OS (108.4 vs 30.0 weeks) relative to GCB subtype patients. Thus, GEP-defined subtypes provided superior predictive power relative to $\mathrm{IHC}$. 
Table 1. Outcomes of historical control patients treated with R-CHOP and study patients treated with $\mathrm{R}^{2}$-CHOP based on GCB and non-GCB DLBCL subtypes.

\begin{tabular}{|c|c|c|c|c|}
\hline \multirow{2}{*}{$\begin{array}{l}\text { Subtype, } \\
\text { treatment }\end{array}$} & \multicolumn{2}{|c|}{ PFS, \% (95\% Cl) } & \multicolumn{2}{|c|}{ OS, \% (95\% Cl) } \\
\hline & 12 months & 24 months & 12 months & 24 months \\
\hline \multicolumn{5}{|l|}{ GCB: } \\
\hline$-\mathrm{R}-\mathrm{CHOP}$ & $73(62-85)$ & $64(53-78)$ & 90 (82-98) & $74(63-86)$ \\
\hline$-\mathrm{R}^{2}-\mathrm{CHOP}$ & $64(49-84)$ & $59(44-80)$ & $88(77-100)$ & 75 (61-93) \\
\hline \multicolumn{5}{|l|}{ Non-GCB: } \\
\hline$-\mathrm{R}-\mathrm{CHOP}$ & $39(25-62)$ & $28(15-51)$ & $61(45-82)$ & $46(30-69)$ \\
\hline$-\mathrm{R}^{2}-\mathrm{CHOP}$ & $72(55-94)$ & $60(41-87)$ & $95(87-100)$ & $83(67-100)$ \\
\hline
\end{tabular}

Data on lenalidomide in first-line DLBCL used to determine COO [15]. Among the 60 lenawere provided by two Phase II single-arm stud- lidomide-treated patients evaluable for response, ies of lenalidomide plus R-CHOP $\left(\mathrm{R}^{2}-\mathrm{CHOP}\right)$. ORR was $98 \%$, with a CR rate of $80 \%$ and A Mayo Clinic study compared 64 patients with a 24-month OS rate of $78 \%$. Not surprisingly, newly diagnosed DLBCL who were treated with the R-CHOP-treated patients with non-GCB oral lenalidomide (25 mg daily, days 1-10) com- disease experienced poorer 24-month PFS (28 vs bined with standard R-CHOP21 with a con- 64\%) and 24-month OS rates (46 vs 74\%) than temporaneously treated, case-matched group of those with the GCB subtype. In contrast, among 87 patients treated outside the study with con- patients treated with $\mathrm{R}^{2}$-CHOP, both 24-month ventional R-CHOP [33]. IHC subtyping was PFS (60 vs 59\%) and OS rates (83 vs 75\%) were

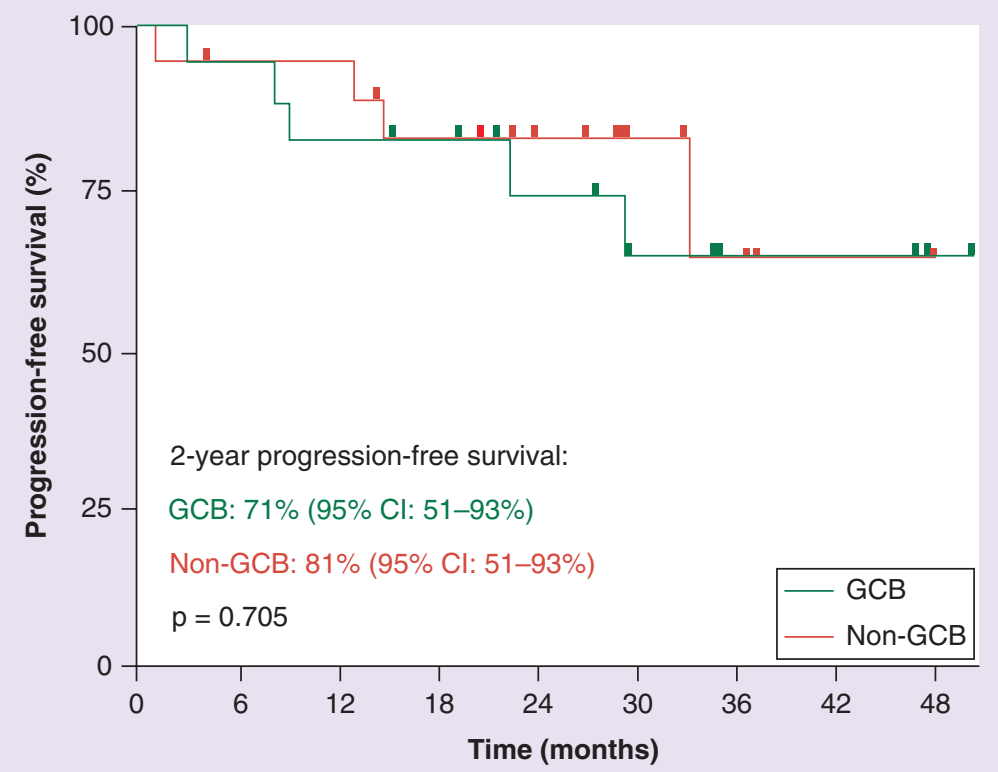

Number at risk

$\begin{array}{rrrrrrrrrr}\text { GCB } & 16 & 14 & 12 & 11 & 8 & 6 & 3 & 3 & 2 \\ \text { Non-GCB } & 16 & 15 & 15 & 12 & 10 & 5 & 3 & 3 & 1\end{array}$

Figure 2. Progression-free survival by cell-of-origin in the Fondazione Italiana Linfomi REAL07 study.

GCB: Germinal center B cell.

Reprinted with permission from [34] @ Elsevier (2014). 
similar for both COO subtypes (Table 1), indicating improved survival for patients with nonGCB disease treated with $\mathrm{R}^{2}$-CHOP compared with those treated with R-CHOP.

In the multicenter FIL REAL07 study, 49 patients aged 64-71 years with newly diagnosed DLBCL or grade $3 \mathrm{~b}$ follicular lymphoma received $15 \mathrm{mg}$ oral lenalidomide on days $1-14$ of six standard cycles of R-CHOP21 [34]. Subtyping was performed by IHC, and final response was assessed by PET scan. The ORR was $92 \%$ (86\% $\mathrm{CR})$. Once again, patients with the non-GCB subtype had PFS similar to those with GCB as the cell-of-origin (Figure 2). The 2-year PFS rates were 81 and $71 \%$ in these subgroups, respectively.

In both first-line $\mathrm{R}^{2}$-CHOP studies, grade 3 or 4 adverse events (AEs) were primarily hematologic and consistent with toxicities expected for R-CHOP alone. The most common grade 3 or 4 adverse events were neutropenia (88 and $79 \%$ ), leukopenia (80 and 59\%) and thrombocytopenia (44 and 30\%) in the Mayo Clinic and FIL studies, respectively [33,34]. In both studies, nonhematologic adverse events were infrequent. In the Mayo Clinic study, nonhematologic AEs were seen in $25 \%$ of patients, with two patients (3\%) experiencing a grade 4 event (one sepsis, one intra-abdominal hemorrhage) and one (2\%) experiencing grade 5 sepsis. In the FIL study, the most common nonhematologic AEs were neurological (4\%) and deep vein thrombosis (4\%). One patient $(2 \%)$ taking cotrimoxazole discontinued lenalidomide due to grade 3 skin rash.

A recent meta-analysis of lenalidomide studies in first- and second-line DLBCL that included data from these two trials found no significant difference in outcomes between lenalidomidetreated patients with GCB and non-GCB disease, concluding that the addition of lenalidomide to R-CHOP appears to mitigate the unfavorable effects of non-GCB phenotype on outcome [35]. Thus, clinical evidence suggests that the $\mathrm{R}^{2}$-CHOP regimen warrants further follow-up in first-line DLBCL. In addition, the exploratory results from the DLC-001 study showing greater improvements in outcomes for patients with the ABC subtype (determined by genome-wide GEP) compared with the less specific non-GCB subtype (determined by IHC) needed to be taken into account in designing subsequent DLBCL studies. The development of a validated 'gene signature' assay capable of rapidly distinguishing between $\mathrm{ABC}$ and GCB subtypes on the basis of a limited number of genes and using FFPE tissue could provide a practical alternative for assuring accurate subtype designation. Such an assay should allow for appropriate patient selection and a more straightforward interpretation of trial results. With this in mind, a large, randomized study of $\mathrm{R}^{2}-\mathrm{CHOP}$ versus placebo plus standard R-CHOP chemotherapy in patients with newly diagnosed ABC subtype DLBCL was designed.

\section{ROBUST}

- Study objectives

The primary objective of ROBUST (DLC-002; NCT02285062), a randomized, double-blind, placebo-controlled, global, Phase III study, is to evaluate the efficacy of $\mathrm{R}^{2}$-CHOP versus

Table 2. Key ROBUST inclusion and exclusion criteria.

\begin{tabular}{|c|c|}
\hline Inclusion criteria & Exclusion criteria \\
\hline $\begin{array}{l}\text { Previously untreated, histologically confirmed } A B C \text {-type }{ }^{\dagger} \text { CD20 } 0^{+} \text {DLBCL } \\
\text { Measurable disease } \geq 1.5 \mathrm{~cm} \text { in the longest diameter and in } \\
2 \text { perpendicular directions by } C T / M R I \\
\text { Age, } 18-80 \text { years }{ }^{\ddagger} \\
\text { International Prognostic Index score } \geq 2 \\
\text { Absolute neutrophil count } \geq 1.5 \times 10^{9} /\left.\right|^{\S} \\
\text { Platelet count } \geq 75 \times 10^{9} / l^{\Uparrow} \\
\text { Hemoglobin } \geq 7.5 \mathrm{~g} / \mathrm{dl} \\
\text { Calculated creatinine clearance } \geq 30 \mathrm{ml} / \mathrm{min} \\
\text { Contraception as appropriate }\end{array}$ & $\begin{array}{l}\text { Unclassifiable or GCB-type DLBCL } \\
\text { History of other malignancies, unless disease free for } \geq 5 \text { years } \\
\text { Evidence of transformed NHL or composite DLBCL/FL } \\
\text { Left ventricular ejection fraction }<45 \% \\
\text { Grade } \geq 2 \text { peripheral neuropathy }\end{array}$ \\
\hline \multicolumn{2}{|c|}{$\begin{array}{l}{ }^{+} \text {As determined by the validated gene-expression profiling assay performed on the NanoString nCounter Analysis System and assessed by central pathology. } \\
{ }^{\ddagger} \text { At investigator's discretion, patients over } 80 \text { years are eligible if their Eastern Cooperative Oncology Group performance status } \leq 1 \text {, each organ system score is } \leq 2 \text { using the } \\
\text { modified cumulative illness rating scale for comorbidity, and if they are otherwise eligible for R-CHOP (rituximab plus cyclophosphamide, doxorubicin, vincristine and } \\
\text { prednisone) per local practice. } \\
\text { 'Unless secondary to bone marrow involvement, where limit is } \geq 1.0 \times 10^{9} / \mathrm{l} \text {. } \\
\text { 'Unless secondary to bone marrow involvement, where limit is } \geq 50 \times 10^{\%} / \text {. } \\
\text { ABC: Activated B cell; CT: Computed tomography; DLBCL: Diffuse large B-cell lymphoma; FL: Follicular lymphoma; GCB: Germinal center B cell; NHL: Non-Hodgkin } \\
\text { lymphoma. }\end{array}$} \\
\hline
\end{tabular}


placebo-R-CHOP in patients with previously untreated ABC-type DLBCL as determined by GEP. The secondary study objective is to compare the safety of $\mathrm{R}^{2}$-CHOP versus placebo-R-CHOP in this patient population.

Exploratory objectives are to compare the time to second progression after randomization (PFS2), to explore genetic mutations in patients receiving $\mathrm{R}^{2}$-CHOP versus placebo-R-CHOP, to explore minimal residual disease (MRD) and clonal heterogeneity/succession in patients receiving $\mathrm{R}^{2}$ CHOP versus placebo-R-CHOP who achieve $\mathrm{CR}$, to investigate molecular markers related to lenalidomide's mechanism of action, and to evaluate the pharmacokinetics (PK) of lenalidomide when coadministered with and without R-CHOP.

\section{- Study end points}

The primary end point is PFS. Secondary end points are event-free survival, OS, CR rate, duration of CR, time to next lymphoma therapy, ORR and health-related quality of life as measured by the EuroQuol 5 Dimension Questionnaire (EQ-5D) [36] and the Functional Assessment of Cancer Therapy for Patients with Lymphoma (FACT-Lym) [37] standardized measures of health status.

Exploratory end points are PFS2, correlation of MRD status with clinical outcomes such as
PFS and OS, and sensitivity and specificity of the MRD next-generation sequencing test, correlation of pretreatment levels of molecular markers with clinical outcome, incidence of mutations of selected genes and correlation with clinical outcome and PK parameters of lenalidomide.

\section{- Key eligibility criteria}

All patients must provide written informed consent. Other inclusion criteria are shown in Table 2.

In ROBUST, DLBCL subtype is determined at screening using an investigational assay based on the previously described Lymph2Cx assay (NanoString Technologies, Inc., Seattle, WA, USA) [38]. A central pathology laboratory in Europe or the USA evaluates FFPE lymph node or tumor samples obtained through surgical incision or excision biopsy or core needle biopsy [39]. Central pathology review of diagnosis, subtype and CD20 + status is completed and the results transmitted within 3 calendar days (inclusive of weekends and holidays) of sample receipt, thus minimizing treatment delays.

\section{- Study design \& treatment}

Approximately 560 eligible patients will be randomized 1:1 to receive $\mathrm{R}^{2}$-CHOP or placebo$\mathrm{R}-\mathrm{CHOP}$ (Figure 3). Randomization is via the

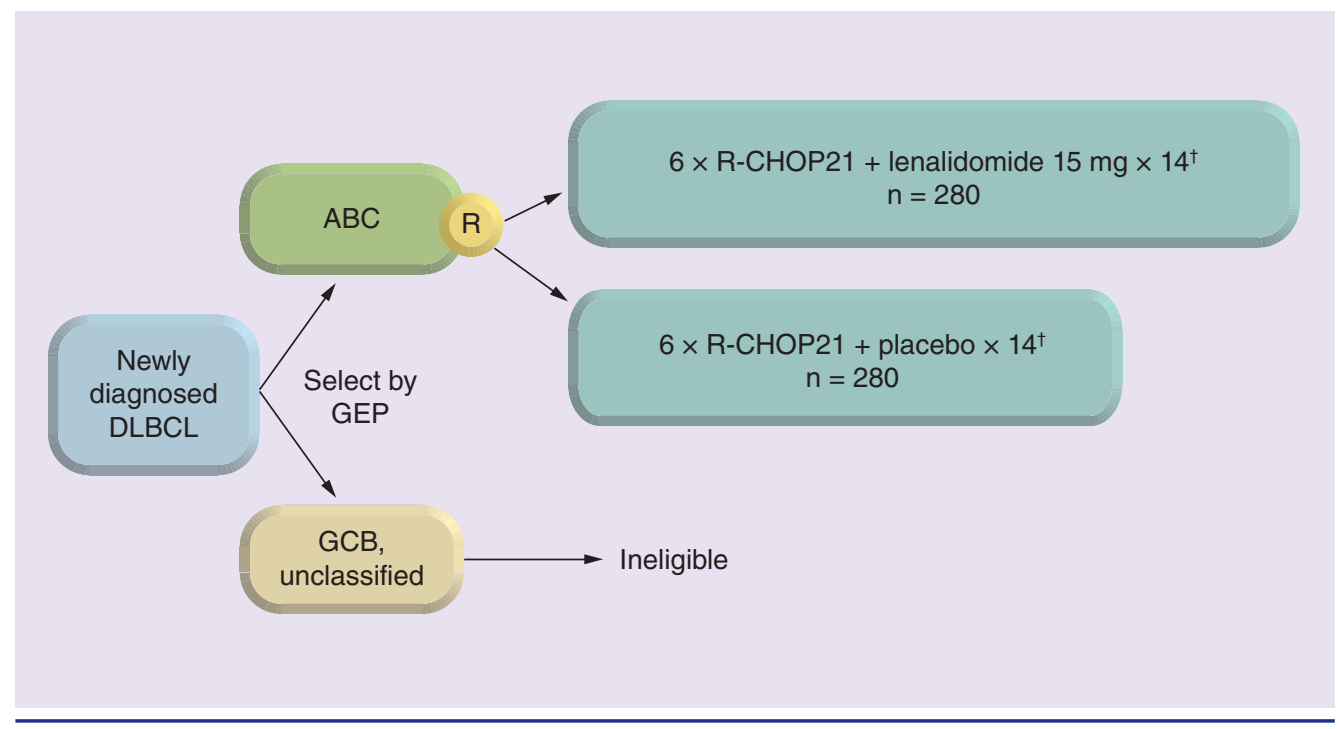

Figure 3. ROBUST study design.

${ }^{+}$Option for two additional rituximab doses after completing treatmentregimen (if considered standard of care per local practice), and option for pre-specified local radiotherapy for bulky disease after study chemotherapy.

ABC: Activated B cell; DLBCL: Diffuse large B-cell lymphoma; GCB: Germinal center B cell; GEP: Geneexpression profiling; R-CHOP: Rituximab plus cyclophosphamide, doxorubicin, vincristine and prednisone. 


\begin{tabular}{|c|c|c|}
\hline Drug & Dose & Dosing days (every 21 days) \\
\hline Lenalidomide/placebo p.o. & $15 \mathrm{mg}$ & $1-14$ \\
\hline Rituximab iv. & $375 \mathrm{mg} / \mathrm{m}^{2}$ & -1 or 1 \\
\hline Cyclophosphamide iv. & $750 \mathrm{mg} / \mathrm{m}^{2}$ & 1 \\
\hline Doxorubicin iv. & $50 \mathrm{mg} / \mathrm{m}^{2}$ & 1 \\
\hline Vincristine iv. & $1.4 \mathrm{mg} / \mathrm{m}^{2}(\mathrm{max} 2.0 \mathrm{mg})$ & 1 \\
\hline Predinsone/prednisolone p.o. & $100 \mathrm{mg}$ & $1-5$ (or day 1 iv. methylprednisone) $^{\dagger}$ \\
\hline
\end{tabular}

interactive voice response system and stratified according to IPI score, presence of bulky disease and age. Patients and investigators are blinded to treatment assignment. The regimens are administered as shown in Table 3. At investigator's discretion prephase treatment with corticosteroids according to local practice is permitted. Patients receive six cycles of treatment, and if standard of care per the local practice, two additional doses of single-agent rituximab may be administered. Treatment continues until completion, unacceptable toxicity, inadequate response to treatment is determined, disease progression, death or withdrawal of consent, whichever occurs first. Primary neutropenia prophylaxis with G-CSF or GM-CSF is mandatory, with dosing and schedule per local practice.

Investigators may prospectively choose to administer local radiotherapy after study chemotherapy for treatment of a particular site of bulky disease (defined as $\geq 7 \mathrm{~cm}$ ). Follow-up begins upon early treatment discontinuation or after six cycles of treatment. Patients are followed for first and second progression, subsequent antilymphoma therapies, development of second primary malignancies and OS.

\section{- Evaluations}

Efficacy is evaluated per the International Working Group Response Criteria for NHL [40],

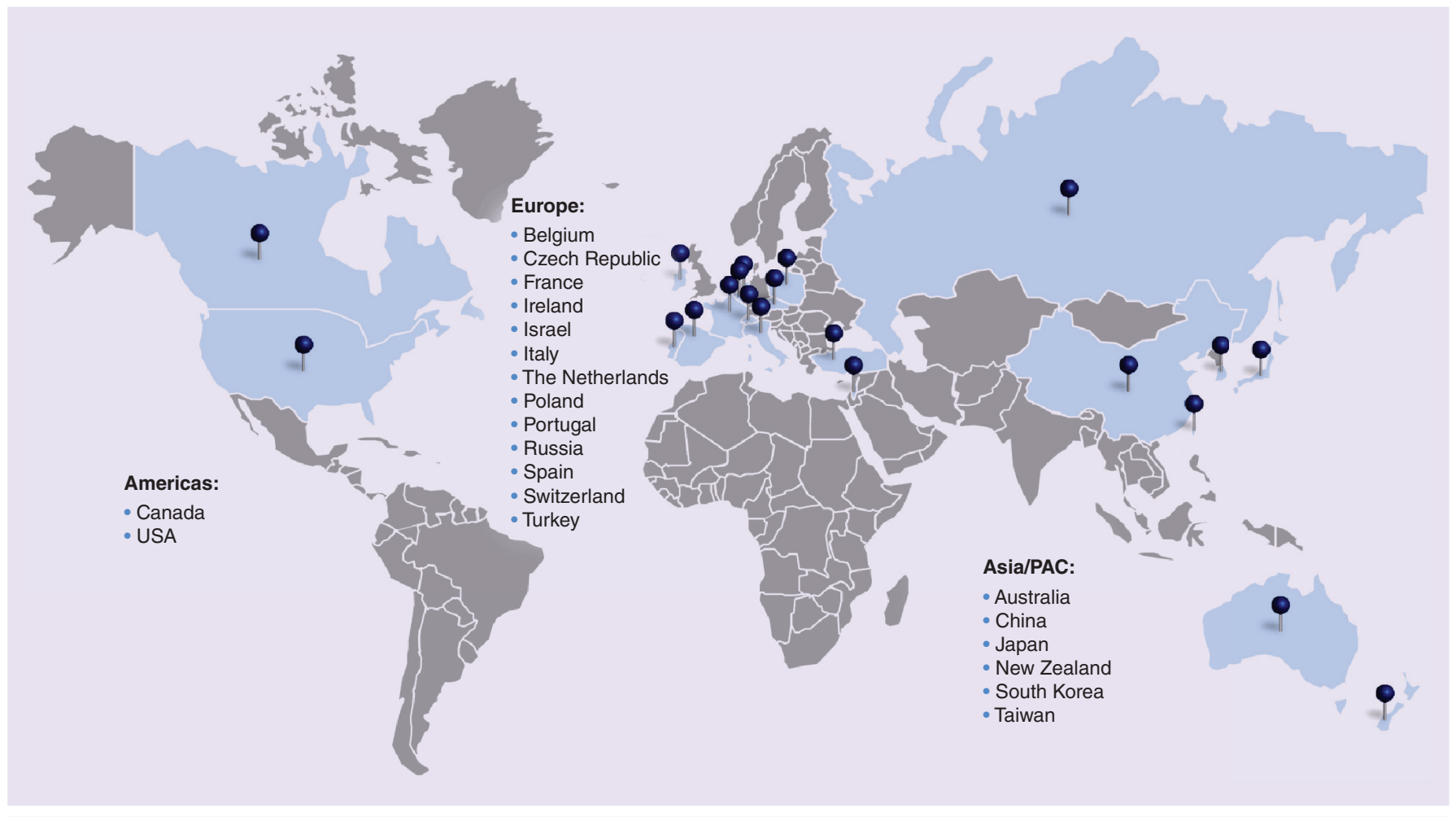

Figure 4. ROBUST participating countries as of February 2016.

PAC: Pacific. 
and PET scans are interpreted according to the Deauville criteria [41]. CT scans are done at screening, midcycle, end of treatment and at 12-week intervals during years 1-3 of the 5-year follow-up period. PET scans are done at screening and at the end of treatment. MRD is assessed using next-generation sequencing, and sample collection is matched to the CT scan assessment schedule. Throughout the study, investigators perform real-time efficacy assessments. Realtime end-of-treatment assessment and batched review of follow-up scans are performed by central radiology. For the primary analysis, PFS events are determined by central review. Quality of life assessment via patient-completed EQ-5D and FACT-Lym instruments is done at screening, after cycles 3 and 6 and every 12 weeks during follow-up, beginning with week 34 .

Safety, including the occurrence of second primary malignancies as events of interest, is monitored throughout the study. Adverse events are coded according to the Medical Dictionary for Regulatory Affairs and are graded according to the National Cancer Institute Common Terminology Criteria for Adverse Events, version 4.0 .

\section{- Statistical considerations}

The intent-to-treat population is defined as all patients who are randomized into the trial, regardless of whether they received study treatment. The safety population is defined as all patients who have received at least one dose of study drug. The primary end point, PFS, will be analyzed in the intent-to-treat population. The safety population will be used for all safety analyses.

At the predetermined sample size (560 patients) ROBUST has a $90 \%$ power to detect a hazard ratio (HR) of 0.625 , that is, a $37.5 \%$ reduction in the PFS HR, at a two-sided 0.05 significance level. Median PFS in the control arm is assumed to be approximately 24 months. An interim analysis for futility is preplanned. Kaplan-Meier estimates of survival will be calculated for time-to-event end points. A Cox proportional hazard model will be used to estimate HRs with 95\% CIs. Treatment effects, however, will be determined by the $\mathrm{p}$-value from the stratified log-rank test.

\section{- Study status}

ROBUST is an international study, with centers located in North America, Europe, Asia and Australia (Figure 4). A list of study centers can be found on the ClinicalTrials.gov website [42]. Enrollment was initiated in January 2015, with expected accrual completion in October 2017. This study is sponsored by Celgene and is a joint scientific collaboration among Fondazione Italiana Linfomi, Mayo Clinic, and Celgene. Further information is available at ClinicalTrials.gov (NCT02285062).

\section{Conclusion}

Recent years bear witness to continued advances in the treatment of hematologic malignancies. In the case of DLBCL, the addition of the anti-CD20 monoclonal antibody rituximab to CHOP was found to significantly improve median PFS and improve median OS in elderly patients with previously untreated disease [43], leading to the widespread adoption of rituximab in standard DLBCL regimens [44]. Using GEP, researchers began to unravel the molecular basis for the divergent outcomes associated with the single disease of DLBCL, identifying a major subtype, $\mathrm{ABC}$, which is associated with poorer survival [12]. Assays for the DLBCL-COO subtypes of GCB and non-GCB using IHC became amenable for routine use, initially of value for predicting prognosis and subsequently for identifying the non-GCB cases associated with inferior survival as candidates for alternative therapies. The demonstration of single-agent activity of lenalidomide in relapsed DLBCL and its mechanism of action that differed from those of other agents in R-CHOP paved the way for the development of the $\mathrm{R}^{2}-\mathrm{CHOP}$ regimen. Indeed, the addition of the immunomodulatory agent lenalidomide to standard first-line $\mathrm{R}-\mathrm{CHOP}$ ranks among the most promising new regimens to date, providing outcomes for patients with non-GCB disease similar to those of patients with the GCB-COO in two Phase II studies $[33,34]$. Discrepancies between the classifications provided by IHC subtyping and those obtained using the 'gold standard' GEP method and the need for confirmatory results in a large, randomized trial remain. ROBUST will address both of these important issues. Rapid COO classification by a validated companion diagnostic GEP assay using routinely available FFPE biopsy tissue will improve the speed and accuracy of COO classifications and allow inclusion of only $\mathrm{ABC}$ patients, the group hypothesized to derive the greatest benefit from $\mathrm{R}^{2}$-CHOP therapy. Increasing the accuracy of routine clinical DLBCL molecular subtyping is an important 


\section{EXECUTIVE SUMMARY}

\section{Diffuse large B-cell lymphom subtypes}

- The ability to classify diffuse large B-cell lymphoma (DLBCL) by cell-of-origin (COO) led to the identification of the activated $B$ cell $(A B C)$ subtype and its association with poor survival relative to the germinal center B-cell (GCB) subtype.

- The original 'gold standard' assay for DLBCL using genome-wide gene-expression profiling (GEP) was unsuitable for routine clinical use; instead, immunohistochemistry $(\mathrm{IHC})$, with a higher rate of misclassification, was generally employed to ascertain GCB versus non-GCB subtypes.

- The Lymph2Cx assay, a novel 20-gene GEP assay that is highly concordant with the 'gold standard' assay for DLBCL and enables analysis of formalin-fixed, paraffin-embedded biopsy samples with a turnaround of 3 calendar days, was developed to overcome limitations of IHC classifiers.

\section{Lenalidomide in DLBCL}

- Lenalidomide is an oral immunomodulatory drug that has antineoplastic activity, as well as indirect effects mediated by immune cells present in the tumor microenvironment.

- The DLC001 trial comparing lenalidomide monotherapy with investigator's choice monotherapy in pretreated DLBCL found significantly improved progression-free survival (PFS) in the lenalidomide arm. Exploratory analysis in lenalidomide-treated patients found improvements in response and survival in patients with the $A B C$ subtype relative to the GCB group.

- In two Phase II, single-arm studies of previously untreated DLBCL, R²-CHOP (lenalidomide plus rituximab and cyclophosphamide, doxorubicin, vincristine and prednisone [R-CHOP]) provided similar PFS benefits to patients in both non-GCB and GCB subgroups, suggesting more pronounced benefits in the former, which is primarily the $A B C$ subtype.

\section{ROBUST}

- ROBUST is a randomized, double-blind, placebo-controlled, global, Phase III study evaluating the efficacy and safety of $\mathrm{R}^{2}-\mathrm{CHOP}$ versus placebo- $\mathrm{R}-\mathrm{CHOP}$ in patients with previously untreated $A B C$-type $\mathrm{DLBCL}$.

- The primary end point is PFS, as assessed by central review. Secondary end points include event-free survival, overall survival, complete response rate, duration of response, time to next lymphoma treatment, overall response rate and health-related quality of life.

- Key eligibility criteria include previously untreated, histologically confirmed ABC-type CD20+ DLBCL.

- To ensure accurate diagnosis, $\mathrm{COO}$ subtype is determined using a clinical trial assay version of Lymph2Cx, a novel 20-gene GEP assay that analyzes formalin-fixed, paraffin-embedded biopsy samples with a turnaround of 3 calendar days.

- Other inclusion criteria include measurable disease and International Prognostic Index score $\geq 2$.

- Approximately 560 patients will be randomized 1:1 to receive six cycles of $\mathrm{R}^{2}$-CHOP (15 mg oral lenalidomide daily, days 1-14, plus standard R-CHOP21) or to placebo (15 mg daily, days 1-14) plus R-CHOP21.

- Two additional doses of single-agent rituximab may be administered if standard of care per local practice.

- Treatment continues until completion, unacceptable toxicity, inadequate response, disease progression or withdrawal of consent.

- The study has a $90 \%$ power to detect a hazard ratio of 0.625 at a two-sided significance level of 0.05 .

- An interim analysis for futility is planned at $50 \%$ of events.

- ROBUST is enrolling at centers in North America, Europe, Asia and Australia.

- A list of study centers can be found at [42].

- Enrollment began in January 2015, with accrual expected to be completed in October 2017. 
step in the evolution of better therapies for patients with DLBCL and a prerequisite for the advancement of precision medicine [21].

\section{Financial \& competing interests disclosure}

This study is supported by Celgene Corporation, Summit, NJ, USA. G Nowakowski received research funding and travel expenses from and has a consultingladvisory role at Celgene. A Chiappella has participated in speaker's bureaus from Celgene, Roche, Pfizer, Janssen and Teva. T Witzig received research funding from Celgene. $M$ Spina received honoraria from Teva, Servier, Janssen-Cilag, Gilead, Roche, Mundipharma and Menarini, has had consulting or advisory roles with Teva, Gilead, Takeda, Janssen and Menarini, has received research funding from Mundipharma, Teva and Menarini, and has received travel expenses from Janssen, Servier, Teva, Menarini, Mundipharma, Gilead, Roche and Takeda. RD Gascoyne receives consulting fees from Celgene, AbbVie and Seattle Genetics, honoraria from Celgene, AbbVie and Seattle
Genetics, and has participated in a Seattle Genetics speaker's bureau. L Zhang, J Flament, and J Repici are employed by Celgene and report Celgene stock ownership. U Vitolo received research funding from Celgene and Roche, has had consulting or advisory roles at Roche and Janssen, and has participated in speaker's bureaus from Roche, Celgene and Takeda. The authors have no other relevant affiliations or financial involvement with any organization or entity with a financial interest in or financial conflict with the subject matter or materials discussed in the manuscript apart from those disclosed.

Medical writing support was provided by Robert Rydzewski MS, CMPP, of Bio Connections LLC, funded by Celgene Corporation.

\section{Open access}

This work is licensed under the AttributionNonCommercial-NoDerivatives 4.0 Unported License. To view a copy of this license, visit http://creativecommons.org/ licenses/by-nc-nd/4.0/

\section{References}

Papers of special note have been highlighted as:

- of interest; $\bullet$ of considerable interest

1 Bachy E, Salles G. Treatment approach to newly diagnosed diffuse large B-cell lymphoma. Semin. Hematol. 52(2), 107-118 (2015).

2 Friedberg JW, Fisher RI. Diffuse large B-cell lymphoma. Hematol. Oncol. Clin. North Am. 22(5), 941-952, ix (2008).

3 Vaidya R, Witzig TE. Prognostic factors for diffuse large B-cell lymphoma in the $\mathrm{R}(\mathrm{X})$ CHOP era. Ann. Oncol. 25(11), 2124-2133 (2014).

4 A clinical evaluation of the International Lymphoma Study Group classification of nonHodgkin's lymphoma. The non-Hodgkin's lymphoma classification project. Blood 89(11), 3909-3918 (1997).

5 Harris NL, Jaffe ES, Stein $\mathrm{H}$ et al. A revised European-American classification of lymphoid neoplasms: a proposal from the International Lymphoma Study Group. Blood 84(5), 1361-1392 (1994).

6 Grogan TM, Lippman SM, Spier CM et al. Independent prognostic significance of a nuclear proliferation antigen in diffuse large cell lymphomas as determined by the monoclonal antibody Ki-67. Blood 71(4), 1157-1160 (1988).

7 Yoon DH, Choi DR, Ahn HJ et al. Ki-67 expression as a prognostic factor in diffuse large B-cell lymphoma patients treated with rituximab plus CHOP. Eur. J. Haematol. 85(2), 149-157 (2010).
8 A predictive model for aggressive nonHodgkin's lymphoma. The International Non-Hodgkin's Lymphoma Prognostic Factors Project. N. Engl. J. Med. 329(14), 987-994 (1993).

9 Sehn LH, Berry B, Chhanabhai M et al. The revised International Prognostic Index (R-IPI) is a better predictor of outcome than the standard IPI for patients with diffuse large B-cell lymphoma treated with R-CHOP. Blood 109(5), 1857-1861 (2007).

10 Zhou Z, Sehn LH, Rademaker AW et al. An enhanced International Prognostic Index (NCCN-IPI) for patients with diffuse large B-cell lymphoma treated in the rituximab era. Blood 123(6), 837-842 (2014).

11 Shipp MA, Abeloff MD, Antman KH et al. International consensus conference on high-dose therapy with hematopoietic stem cell transplantation in aggressive nonHodgkin's lymphomas: report of the jury. J. Clin. Oncol. 17(1), 423-429 (1999).

12 Alizadeh AA, Eisen MB, Davis RE et al. Distinct types of diffuse large B-cell lymphoma identified by gene expression profiling. Nature 403(6769), 503-511 (2000).

-. The authors used the 'gold standard', gene-expression profiling, to identify distinct subtypes of diffuse large B-cell lymphoma (DLBCL) on the basis of cell-of-origin ( $\mathrm{COO})$, germinal center $\mathrm{B}$ cell (GCB) or activated B cell (ABC).

13 Rosenwald A, Wright G, Chan WC et al. The use of molecular profiling to predict survival after chemotherapy for diffuse large-B-cell lymphoma. N. Engl. J. Med. 346(25), 1937-1947 (2002).

- Using GEP analysis of 240 DLBCL biopsy samples, a 17 -gene predictor of overall survival was constructed, and data confirmed the poorer outcomes associated with $A B C$ and type 3 subtypes relative to the GCB subtype.

14 Pasqualucci L, Dalla-Favera R. The genetic landscape of diffuse large B-cell lymphoma. Semin. Hematol. 52(2), 67-76 (2015).

15 Hans CP, Weisenburger DD, Greiner TC et al. Confirmation of the molecular classification of diffuse large B-cell lymphoma by immunohistochemistry using a tissue microarray. Blood 103(1), 275-282 (2004).

- An immunohistochemistry method that uses only three immunostains and was capable of distinguishing GCB and non-GCB subtypes of DLBCL was developed and has subsequently received wide clinical use.

16 Nowakowski GS, Macon WR, Gascoyne RD, Witzig TE. Reply to M. Gleeson et al. J. Clin. Oncol. 33(28), 3216-3217 (2015).

17 Gutierrez-Garcia G, Cardesa-Salzmann T, Climent F et al. Gene-expression profiling and not immunophenotypic algorithms predicts prognosis in patients with diffuse large B-cell lymphoma treated with immunochemotherapy. Blood 117(18), 4836-4843 (2011).

18 Scott DW, Wright GW, Williams PM et al. Determining cell-of-origin subtypes of diffuse 
large B-cell lymphoma using gene expression in formalin-fixed paraffin-embedded tissue. Blood 123(8), 1214-1217 (2014).

-• A 20-gene gene-expression profiling assay using formalin-fixed, paraffin-embedded biopsy samples that robustly assigns cell-of-origin subtypes of DLBC correctly assigned more than $95 \%$ of samples with low interlaboratory variability and is used as the companion diagnostic in ROBUST.

19 Lenz G, Wright G, Dave SS et al. Stromal gene signatures in large-B-cell lymphomas. N. Engl. J. Med. 359 (22), 2313-2323 (2008).

20 Scott DW, Mottok A, Ennishi D et al. Prognostic significance of diffuse large B-cell lymphoma cell-of-origin determined by digital gene expression in formalin-fixed paraffin-embedded tissue biopsies. J. Clin. Oncol. 33(26), 2848-2856 (2015).

21 Jameson JL, Longo DL. Precision medicinepersonalized, problematic, and promising. N. Engl. J. Med. 372(23), 2229-2234 (2015).

22 Gribben JG, Fowler N, Morschhauser F. Mechanisms of action of lenalidomide in B-cell non-Hodgkin lymphoma. J. Clin. Oncol. 33(25), 2803-2811 (2015).

23 Lopez-Girona A, Mendy D, Ito T et al. Cereblon is a direct protein target for immunomodulatory and antiproliferative activities of lenalidomide and pomalidomide. Leukemia 26(11), 2326-2335 (2012).

24 Wu L, Adams M, Carter T et al. Lenalidomide enhances natural killer cell and monocyte-mediated antibody-dependent cellular cytotoxicity of rituximab-treated CD20+ tumor cells. Clin. Cancer Res. 14(14), 4650-4657 (2008).

25 Zhang L, Qian Z, Cai Z et al. Synergistic antitumor effects of lenalidomide and rituximab on mantle cell lymphoma in vitro and in vivo. Am. J. Hematol. 84(9), 553-559 (2009).

26 REVLIMID ® (lenalidomide) prescribing information. Summit, NJ, USA, Celgene Corporation (2015).

27 Zhang LH, Kosek J, Wang M, Heise C, Schafer PH, Chopra R. Lenalidomide efficacy in activated B-cell-like subtype diffuse large B-cell lymphoma is dependent upon IRF4 and cereblon expression. Br. J. Haematol. 160(4), 487-502 (2013).

28 Wiernik PH, Lossos IS, Tuscano JM et al. Lenalidomide monotherapy in relapsed or refractory aggressive non-Hodgkin's lymphoma. J. Clin. Oncol. 26(30), 4952-4957 (2008).

29 Witzig TE, Vose JM, Zinzani PL et al. An international Phase II trial of single-agent lenalidomide for relapsed or refractory aggressive B-cell non-Hodgkin's lymphoma. Ann. Oncol. 22(7), 1622-1627 (2011).

30 Czuczman MS, Vose J, Zinzani PL et al. Lenalidomide monotherapy is clinically active in patients with relapsed/refractory diffuse large B-cell lymphoma (DLBCL): a pooled analysis of data from 2 Phase II studies (NHL-002/003). Haematologica 95(Suppl. 2), 239, Abstract 0574 (2010).

31 Hernandez-Ilizaliturri FJ, Deeb G, Zinzani PL et al. Higher response to lenalidomide in relapsed/refractory diffuse large B-cell lymphoma in nongerminal center B-cell-like than in germinal center B-cell-like phenotype. Cancer 117(22), 5058-5066 (2011).

- A retrospective analysis of relapsed/ refractory DLBCL studies that found higher overall response and complete response rates to lenalidomide, along with longer progression-free survival in non-GCB disease relative to the GCB subtype.

32 Czuczman MS, Davies A, Linton KM et al. A Phase 2/3 multicenter, randomized study comparing the efficacy and safety of lenalidomide versus investigator's choice in relapsed/refractory DLBCL. Blood 124, 628 (2014).

- This abstract report results from the DLC001 trial of lenalidomide versus investigator's choice monotherapy in relapsed/refractory DLBCL, showing improved outcomes in the lenalidomide arm, with particular benefit in patients with the $\mathrm{ABC}$ subtype.

33 Nowakowski GS, Laplant B, Macon WR et al. Lenalidomide combined with R-CHOP overcomes negative prognostic impact of non-germinal center $\mathrm{B}$-cell phenotype in newly diagnosed diffuse large B-cell lymphoma: a Phase II study. J. Clin. Oncol. 33(3), 251-257 (2015).

-• One of two Phase II studies of $\mathrm{R}^{2}$-CHOP in previously untreated DLBCL, this study demonstrates superior outcomes in the $\mathrm{ABC}$ subtype group relative to a historical control group treated with R-CHOP.

34 Vitolo U, Chiappella A, Franceschetti $\mathrm{S}$ et al. Lenalidomide plus R-CHOP21 in elderly patients with untreated diffuse large B-cell lymphoma: results of the REAL07 open-label, multicentre, Phase 2 trial. Lancet Oncol. 15(7), 730-737 (2014).

-• The REAL07 single-arm, Phase II trial of $\mathrm{R}^{2}$-CHOP in elderly patients with previously untreated DLBCL found similar progression-free survival benefit in both GCB and non-GCB subtypes.
35 Fang C, Zhu D, Dong $\mathrm{H}$ et al. Lenalidomide alone or in combination with chemotherapy treatment for subtypes of diffuse large B cell lymphoma: a systematic review and meta-analysis. Int. J. Clin. Exp. Med. 8(7), 10705-10713 (2015).

36 Pickard AS, Wilke CT, Lin HW, Lloyd A. Health utilities using the EQ-5D in studies of cancer. Pharmacoeconomics 25(5), 365-384 (2007).

37 Hlubocky FJ, Webster K, Cashy J, Beaumont J, Cella D. The development and validation of a measure of health-related quality of life for non-Hodgkin's lymphoma: The Functional Assessment of Cancer Therapy-Lymphoma (FACT-Lym). Lymphoma 54(9), 1942-1946 (2013).

38 Wallden B, Ferree S, Ravi $\mathrm{H}$ et al. Development of the molecular diagnostic (MDX) DLBCL lymphoma subtyping test (LST) on the Ncounter analysis system. J. Clin. Oncol. 33(Suppl. 15), Abstract 8536 (2015).

39 Storhoff J, Wallden B, Braziel RM et al. Analytical performance of Nanostring's Companion Diagnostic (CDx) Lymphoma Subtyping Test (LST) with diffuse large B-cell lymphoma core needle biopsy samples. Blood 126(23), Abstract 1481 (2015).

40 Cheson BD, Fisher RI, Barrington SF et al. Recommendations for initial evaluation, staging, and response assessment of Hodgkin and non-Hodgkin lymphoma: the Lugano classification. J. Clin. Oncol. 32(27), 3059-3068 (2014).

41 Itti E, Meignan M, Berriolo-Riedinger A et al. An international confirmatory study of the prognostic value of early PET/CT in diffuse large B-cell lymphoma: comparison between Deauville criteria and Deltasuvmax. Eur. J. Nucl. Med. Mol. Imaging 40(9), 1312-1320 (2013).

42 ClinicalTrials.gov. www.clinicaltrials.gov

43 Feugier P, Van Hoof A, Sebban C et al. Long-term results of the R-CHOP study in the treatment of elderly patients with diffuse large B-cell lymphoma: a study by the Groupe d'Etude des Lymphomes de l'Adulte. J. Clin. Oncol. 23(18), 4117-4126 (2005).

44 Fu K, Weisenburger DD, Choi WW et al. Addition of rituximab to standard chemotherapy improves the survival of both the germinal center B-cell-like and nongerminal center B-cell-like subtypes of diffuse large B-cell lymphoma. J. Clin. Oncol. 26(28), 4587-4594 (2008). 